\title{
Lower Bound of Concurrence Based on Positive Maps
}

\author{
Xiao-Sheng $\mathrm{Li}^{1}$, Xiu-Hong $\mathrm{Gao}^{2}$, and Shao-Ming $\mathrm{Fei}^{2,3}$ \\ ${ }^{1}$ Department of Mathematics, School of Science, \\ South China University of Technology, Guangzhou 510640, China \\ ${ }^{2}$ School of Mathematical Sciences, Capital Normal University, Beijing 100048, China \\ ${ }^{3}$ Max-Planck-Institute for Mathematics in the Sciences, 04103 Leipzig, Germany
}

\begin{abstract}
We study the concurrence of arbitrary dimensional bipartite quantum systems. An explicit analytical lower bound of concurrence is obtained, which detects entanglement for some quantum states better than some well-known separability criteria, and improves the lower bounds such as from the PPT, realignment criteria and the Breuer's entanglement witness.
\end{abstract}

PACS numbers: 03.67.Mn, 03.67.-a, 02.20.Hj, 03.65.-w 
Quantum entangled states are the most important resource in quantum information processing [1]. An important theoretical challenge in the theory of quantum entanglement is to give a proper description and quantification of quantum entanglement for given quantum states. The entanglement of formation (EOF) [2] and concurrence [3] are two well defined quantitative measures of quantum entanglement. For the two-qubit case EOF is a monotonically increasing function of the concurrence and an elegant formula for the concurrence was derived analytically by Wootters in [4]. It plays an essential role in describing quantum phase transitions in various interacting quantum many-body systems [5, 6] and can be experimentally measured [7]. However for general high dimensional case, due to the extremizations involved in the calculation, only a few explicit analytic formulae for EOF and concurrence have been found for some special symmetric states [8].

In stead of analytic formulae, some progresses have been made toward the lower bounds of EOF and concurrence. For instance, in [9] a lower bound of concurrence that can be tightened by numerical optimization over some parameters has been derived. In [10, 11] analytic lower bounds on EOF and concurrence for any dimensional mixed bipartite quantum states have been presented by using the positive partial transposition (PPT) and realignment separability criteria. These bounds are exact for some special classes of states and can be used to detect many bound entangled states. In [12] another lower bound on EOF for even dimensional bipartite states has been presented from a new separability criterion [13]. A lower bound of concurrence based on local uncertainty relations (LURs) criterion is derived in [14]. This bound is further optimized in [15]. In [16, 17] the authors presented lower bounds of concurrence for bipartite systems in terms of a different approach, which has a close relationship with the distillability of bipartite quantum states. In [18] an experimentally measurable bounds for EOF has been presented. All these bounds obtained so far together give rise to a good quantitative estimation of $\mathrm{EOF}$ and concurrence. In particular, they are supplementary in detecting entanglement.

In this brief report, based on positive maps, we present a new lower bound of concurrence for arbitrary dimensional bipartite systems. This bound is shown to detect entanglement that can not be recognized by the bounds in [11, 12].

Let $H_{1}$ and $H_{2}$ be $N$-dimensional vector spaces. A bipartite quantum pure state $|\psi\rangle$ in 
$H_{1} \otimes H_{2}$ has a Schmidt form

$$
|\psi\rangle=\sum_{i} \alpha_{i}\left|e_{i}^{1}\right\rangle \otimes\left|e_{i}^{2}\right\rangle
$$

where $\left|e_{i}^{1}\right\rangle$ and $\left|e_{i}^{2}\right\rangle$ are the orthonormal bases in $H_{1}$ and $H_{2}$ respectively, $\alpha_{i}$ are the Schmidt coefficients satisfying $\sum_{i} \alpha_{i}^{2}=1$.

The concurrence of the state $|\psi\rangle$ is given by

$$
\left.C(|\psi\rangle)=\sqrt{2\left(1-\operatorname{Tr} \rho_{1}^{2}\right.}\right)=2 \sqrt{\sum_{i<j} \alpha_{i}^{2} \alpha_{j}^{2}},
$$

where the reduced density matrix $\rho_{1}$ is obtained by tracing over the second subsystem of the density matrix $\rho=|\psi\rangle\left\langle\psi\left|, \rho_{1}=T r_{2}\right| \psi\right\rangle\langle\psi|$.

A general mixed state in $H_{1} \otimes H_{2}$ has pure state decompositions, $\rho=\sum_{i} p_{i}\left|\psi_{i}\right\rangle\left\langle\psi_{i}\right|$, where $p_{i} \geq 0$ and $\sum_{i} p_{i}=1$. The concurrence is extended to mixed states $\rho$ by the convex roof,

$$
C(\rho)=\min \sum_{i} p_{i} C\left(\left|\psi_{i}\right\rangle\right)
$$

where the minimum is taken over all possible convex decompositions of $\rho$ into an ensemble $\left\{\left|\psi_{i}\right\rangle\right\}$ of pure states with probability distribution $\left\{p_{i}\right\}$.

Let $f(\rho)$ be a real-valued and convex functional on the total state space with the following property,

$$
f(|\psi\rangle\langle\psi|) \leq 2 \sum_{i<j} \alpha_{i} \alpha_{j}
$$

for all state vectors $|\psi\rangle$ with Schmidt decompositions (11). By using the inequality in [11],

$$
\sum_{i<j} \alpha_{i}^{2} \alpha_{j}^{2} \geq \frac{2}{N(N-1)}\left(\sum_{i<j} \alpha_{i} \alpha_{j}\right)^{2},
$$

Breuer [12] has derived that $C(\rho)$ satisfies

$$
C(\rho) \geq \sqrt{\frac{2}{N(N-1)}} f(\rho) .
$$

The $f(\rho)$ corresponding to the lower bounds in [11] are the ones with respect to the PPT criterion and the realignment criterion, $f_{p p t}(\rho)=\left\|\rho^{T_{1}}\right\|-1, f_{r}(\rho)=\|\tilde{\rho}\|-1$, where $\|\cdot\|$ stands for the trace norm of a matrix, $T_{1}$ the partial transposition associated with the space $H_{1}$ and $\tilde{\rho}$ the realigned matrix of $\rho$. While the lower bound in [12] corresponds to a convex functional $f_{W}(\rho)=-\operatorname{Tr}(W \rho)$, where $W$ is the entanglement witness introduced in [12]. 
Let $\Phi$ be a matrix map that maps an $N \times N$ matrix $A,(A)_{i j}=a_{i j}, i, j=1, \ldots N$, to an $N \times N$ matrix $\Phi(A)$ with $(\Phi(A))_{i j}=-a_{i j}$ for $i \neq j$, and $(\Phi(A))_{i i}=(N-2) a_{i i}+a_{i^{\prime} i^{\prime}}$, where $i^{\prime}=i+1 \bmod N$. It can be shown that the matrix map $\Phi$ is positive but not completely positive [19].

Theorem For any bipartite quantum state $\rho=\sum_{i} p_{i}\left|\psi_{i}\right\rangle\left\langle\psi_{i}\right| \in H_{1} \otimes H_{2}$, the concurrence $C(\rho)$ satisfies

$$
C(\rho) \geq \sqrt{\frac{2}{N(N-1)}}\left(\left\|\left(I_{N} \otimes \Phi\right) \rho\right\|-(N-1)\right),
$$

where $I_{N}$ is the $N \times N$ identity matrix.

Proof Set $f(|\psi\rangle\langle\psi|)=\|\left(I_{N} \otimes \Phi\right)|\psi\rangle\langle\psi| \|-(N-1)$. Obviously $f(|\psi\rangle\langle\psi|)$ is convex as the trace norm is convex. What we need to prove is that, for any pure state in Schmidt form (1), the inequality (4) holds.

Since the trace norm does change under local coordinate transformation, we take $|\psi\rangle=$ $\left(\alpha_{1}, 0, \cdots, 0,0, \alpha_{2}, \cdots, 0,0,0, \alpha_{3}, \cdots, 0, \cdots \cdots, 0, \cdots, 0, \alpha_{N}\right)^{t}$, where $t$ denotes transposition, the Schmidt coefficients satisfy $0 \leq \alpha_{1}, \alpha_{2}, \alpha_{3}, \cdots, \alpha_{N} \leq 1, \alpha_{1}^{2}+\alpha_{2}^{2}+\alpha_{3}^{2}+\cdots+\alpha_{N}^{2}=1$.

It is direct to verify that the matrix $T \equiv\left(I_{N} \otimes \Phi\right)(|\psi\rangle\langle\psi|)$ has $N^{2}-2 N$ singular values $0, N$ singular values $\alpha_{1}^{2}, \alpha_{2}^{2}, \alpha_{3}^{2}, \cdots, \alpha_{N}^{2}$, the remaining $N$ ones are the singular values of the following matrix $B$ :

$$
B=\left(\begin{array}{ccccc}
(N-2) \alpha_{1}^{2} & -\alpha_{1} \alpha_{2} & -\alpha_{1} \alpha_{3} & \cdots & -\alpha_{1} \alpha_{N} \\
-\alpha_{1} \alpha_{2} & (N-2) \alpha_{2}^{2} & -\alpha_{2} \alpha_{3} & \cdots & -\alpha_{2} \alpha_{N} \\
\vdots & \vdots & \vdots & \cdots & \vdots \\
-\alpha_{1} \alpha_{N} & -\alpha_{2} \alpha_{N} & -\alpha_{3} \alpha_{N} & \cdots & (N-2) \alpha_{N}^{2}
\end{array}\right) .
$$

As $B$ is Hermitian and real, its singular values are simply given by the square roots of the eigenvalues of $B^{2}$. In fact we only need to consider the absolute values of the eigenvalues of $B$. The eigenpolynomial equation of $B$ is:

$$
\begin{aligned}
H(x)= & \left|x I_{N}-B\right|=x^{N}-(N-2) x^{N-1}+(N-3)(N-1)\left(\sum_{i<j} \alpha_{i}^{2} \alpha_{j}^{2}\right) x^{N-2} \\
& -(N-4)(N-1)^{2}\left(\sum_{i<j<k} \alpha_{i}^{2} \alpha_{j}^{2} \alpha_{k}^{2}\right) x^{N-3}+\cdots \\
& +(-1)^{N}(N-1)^{N-3}\left(\sum_{i_{1}<i_{2} \cdots<i_{N-2}} \alpha_{i_{1}}^{2} \alpha_{i_{2}}^{2} \cdots \alpha_{i_{N-2}}^{2}\right) x^{2} \\
& +(-1)^{N+1}(N-1)^{N-1}\left(\alpha_{1}^{2} \alpha_{2}^{2} \alpha_{3}^{2} \cdots \alpha_{N}^{2}\right)=0 .
\end{aligned}
$$


Let $x_{1}, x_{2}, x_{3}, \cdots, x_{N}$ denote the $N$ roots of Eq.(7). By using the relations between roots and coefficients of the polynomial equation, one has

$$
\sum_{i=1}^{N} x_{i}=N-2, \quad \Pi_{i=1}^{N} x_{i}=(-1)^{2 N+1}(N-1)^{N-1} \Pi_{i=1}^{N} \alpha_{i}^{2} .
$$

The inequality (4) that needs to be proved has the form now,

$$
\sum_{i=1}^{N}\left|x_{i}\right|-(N-2) \leq 2\left(\sum_{i<j} \alpha_{i} \alpha_{j}\right)
$$

where $\sum_{i=1}^{N} \alpha_{i}^{2}=1$ has been taken into account.

To deal with the eigenpolynomial equation (7), we set $\beta=\Pi_{i=1}^{N} \alpha_{i}^{2}$.

(a) If $\beta=0$, then $H(0)=0,0$ is an eigenvalue of $B$. From the derivative of $H(x)$ with respect to $x$,

$$
\begin{aligned}
H^{\prime}(x)= & N x^{N-1}-(N-2)(N-1) x^{N-2}+(N-3)(N-2)(N-1)\left(\sum_{i<j} \alpha_{i}^{2} \alpha_{j}^{2}\right) x^{N-3}(10 \\
& -\cdots+2(-1)^{N}(N-1)^{N-3}\left(\sum_{i_{1}<i_{2} \cdots<i_{N-2}} \alpha_{i_{1}}^{2} \alpha_{i_{2}}^{2} \cdots \alpha_{i_{N-2}}^{2}\right) x
\end{aligned}
$$

we know that if $N$ is even, $H^{\prime}(x)<0$ when $x<0$. Therefore $H(x)$ is a monotonically decreasing function when $x<0$. Taking into account that $H(0)=0$, we see that there exist no negative roots of (7) in this case.

The inequality (9) that needs to be proved has the form now,

$$
\sum_{i=1}^{N} x_{i}-(N-2) \leq 2\left(\sum_{i<j} \alpha_{i} \alpha_{j}\right)
$$

According to the relation in (8), the left hand of the inequality (11) is zero. Hence the inequality (9) is satisfied.

When $N$ is odd, $H(x)$ is a monotonically increasing function for $x<0$. There are also no negative roots of (7). One can similarly prove the inequality (9).

(b) When $\beta \neq 0$, we have $H(0)=(-1)^{N+1}(N-1)^{N-1}\left(\alpha_{1}^{2} \alpha_{2}^{2} \alpha_{3}^{2} \cdots \alpha_{N}^{2}\right)$. If $N$ is even, we have $H(0)<0$. From (8) we get $x_{1} x_{2} x_{3} \cdots x_{N}<0$. Therefore, there exists at least one negative root, say $x_{1}<0$, such that $H\left(x_{1}\right)=0$.

Due to that $H^{\prime}(x)<0$ for $x<0, H(x)$ is a monotonically decreasing function when $x<0$. Taking into account that $H(0)<0$, we have that $x_{1}<0$ is the only negative root. 
Hence the inequality (9) needed to be proved becomes:

$$
\sum_{i=2}^{N} x_{i}-x_{1}-(N-2) \leq 2\left(\sum_{i<j} \alpha_{i} \alpha_{j}\right)
$$

From Eq. (8), we only need to prove that $x_{1} \geq-\sum_{i<j} \alpha_{i} \alpha_{j}$. From the definition of $H(x)$, we have $H\left(-\sum_{i<j} \alpha_{i} \alpha_{j}\right)=\left|-\left(\sum_{i<j} \alpha_{i} \alpha_{j}\right) I_{N}-B\right|=\left|\left(\sum_{i<j} \alpha_{i} \alpha_{j}\right) I_{N}+B\right| \geq 0$, where in the last step the property of the diagonally dominant matrix $\left(\sum_{i<j} \alpha_{i} \alpha_{j}\right) I_{N}+B$ has been used. Since $H\left(x_{1}\right)=0 \leq H\left(-\sum_{i<j} \alpha_{i} \alpha_{j}\right)$ and $H(x)$ is a monotonically decreasing function when $x<0$, we have that $x_{1} \geq-\sum_{i<j} \alpha_{i} \alpha_{j}$.

When $N$ is odd, $H(x)$ is a monotonically increasing function when $x<0$. The theorem can be similarly proved.

Our bound (6) can detect better entanglement than other lower bounds of concurrence. As an example let us consider a state in $4 \times 4$ [19],

$$
\rho=(1 / 4) \operatorname{diag}\left(q_{1}, q_{4}, q_{3}, q_{2}, q_{2}, q_{1}, q_{4}, q_{3}, q_{3}, q_{2}, q_{1}, q_{4}, q_{4}, q_{3}, q_{2}, q_{1}\right)+\frac{q_{1}}{4} \sum_{i, j=1,6,11,16}^{i \neq j} F_{i, j},
$$

where $F_{i, j}$ is the unit matrix with $(i, j)$-entry 1 and others $0, q_{m} \geq 0, \sum q_{m}=1, m=1,2,3,4$.

For $N=4$, the positive map $\Phi$ maps a matrix $M$ with $(M)_{i j}=\left(m_{i j}\right), i, j=1, \ldots, 4$, to

$$
\Phi(M)=\left(\begin{array}{cccc}
2 m_{11}+m_{22} & -m_{12} & -m_{13} & -m_{14} \\
-m_{21} & 2 m_{22}+m_{33} & -m_{23} & -m_{24} \\
-m_{31} & -m_{32} & 2 m_{33}+m_{44} & -m_{34} \\
-m_{41} & -m_{42} & -m_{43} & 2 m_{44}+m_{11}
\end{array}\right) .
$$

By direct computation we have the following set of eigenvalues of $\left(I_{4} \otimes \Phi\right)(\rho)$ :

$$
\begin{gathered}
\frac{1}{4}\left\{q_{1}+2 q_{2}, q_{1}+2 q_{2}, q_{1}+2 q_{2}, q_{1}+2 q_{2}, q_{2}+2 q_{3}, q_{2}+2 q_{3}, q_{2}+2 q_{3}, q_{2}+2 q_{3}\right. \\
\left.q_{3}+2 q_{4}, q_{3}+2 q_{4}, q_{3}+2 q_{4}, q_{3}+2 q_{4}, q_{4}-q_{1}, 3 q_{1}+q_{4}, 3 q_{1}+q_{4}, 3 q_{1}+q_{4}\right\}
\end{gathered}
$$

Therefore from (6]) we have

$$
C(\rho) \geq \sqrt{\frac{1}{6}}\left(\left\|\left(I_{4} \otimes \Phi\right) \rho\right\|-3\right)=\frac{1}{4 \sqrt{6}}\left(q_{1}-q_{4}+\left|q_{1}-q_{4}\right|\right) .
$$

From [11], with respect to the PPT and realignment operation one has bounds

$$
\begin{aligned}
C_{P P T}(\rho) \geq & \sqrt{\frac{1}{6}}\left(\left\|\rho^{T_{1}}\right\|-1\right) \\
= & \frac{1}{2 \sqrt{6}}\left(2 q_{1}+\left|q_{1}-q_{3}\right|+\left|q_{2}+q_{4}-\sqrt{4 q_{1}^{2}+\left(q_{2}-q_{4}\right)^{2}}\right|\right. \\
& \left.+\sqrt{4 q_{1}^{2}+\left(q_{2}-q_{4}\right)^{2}}-1\right)
\end{aligned}
$$


and

$$
\begin{aligned}
C_{r}(\rho) & \geq \sqrt{\frac{1}{6}}(\|\tilde{\rho}\|-1) \\
& =\sqrt{\frac{1}{6}}\left(3 q_{1}+\frac{1}{4}\left(\sqrt{\left(q_{1}-q_{2}+q_{3}-q_{4}\right)^{2}}+2 \sqrt{\left(q_{1}-q_{3}\right)^{2}+\left(q_{2}-q_{4}\right)^{2}}-3\right)\right) .
\end{aligned}
$$

From the formula presented in [12], one has the bound

$$
C_{W}(\rho) \geq \sqrt{\frac{1}{6}}(-\operatorname{tr}(W \rho))=-\frac{1}{2 \sqrt{6}}\left(q_{2}+2 q_{3}+q_{4}\right)
$$

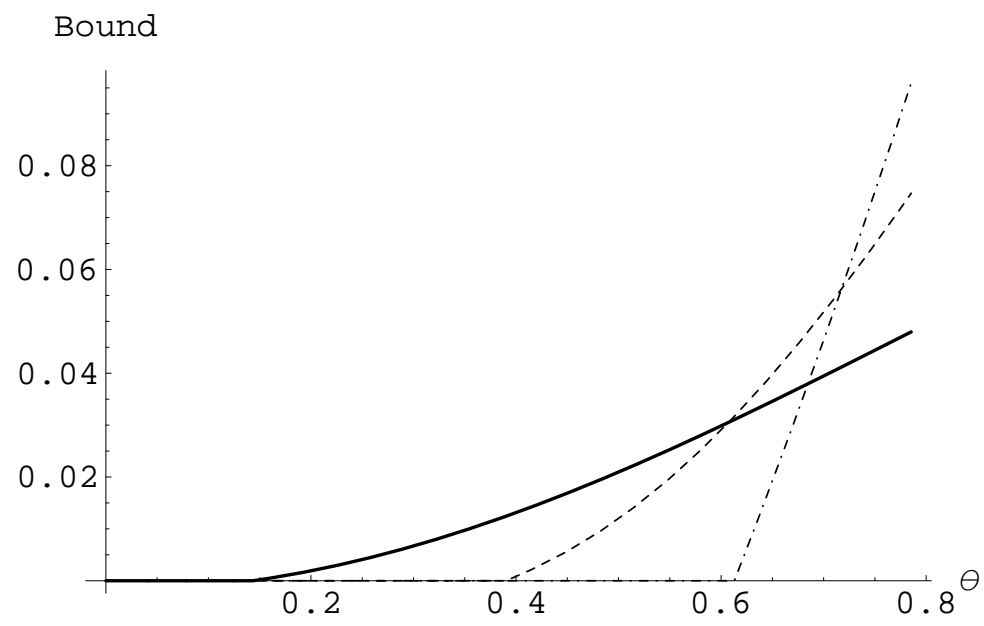

FIG. 1: Lower bounds for the state (13). Solid line: the lower bound given by (14); Dashed line: lower bound given by (15); Dashed-dotted line: lower bound given by (16); $\theta$ axis: lower bound given by (17).

To compare among these bounds, let us take $q_{2}=\frac{1}{2}, q_{4}=0.01, q_{1}=\left(1-q_{2}-q_{4}\right) \sin ^{2} \theta$, $q_{3}=\left(1-q_{2}-q_{4}\right) \cos ^{2} \theta, \theta \in\left[0, \frac{\pi}{4}\right]$. From Fig. 1 we see that our new bound (14) detects entanglement for $\theta>0.143$. While $C_{P P T}$ and $C_{r}$ detect entanglement for $\theta>0.390$ and $\theta>0.613$ respectively, and $C_{W}$ can not detect any entanglement as it is always negative.

In summary, by using a positive map we have presented a new lower bound of concurrence for arbitrary dimensional bipartite systems. By a detailed example we have shown that this bound is better for some states than the lower bounds from the PPT criterion, the realignment criterion and the Breuer's entanglement witness [12] in detecting quantum entanglement.

Acknowledgments This work was supported by the NSFC (10875081,10801100,10871228), KZ200810028013 and PHR201007107. 
[1] R. Horodecki, P. Horodecki, M. Horodecki, and K. Horodecki, Rev. Mod. Phys. 81, 865(2009).

[2] C.H. Bennett, D.P. DiVincenzo and J.A. Smolin, et al. Phys. Rev. A 54, 3824(1996).

M.B. Plenio and S. Virmani, Quant. Inf. Comp. 7, 1(2007).

[3] A. Uhlmann Phys. Rev. A 62, 032307(2000);

P. Rungta, V. Bužek, and C.M. Caves, et al. Phys. Rev. A 64, 042315(2001).

S. Albeverio and S.M. Fei, J. Opt. B: Quantum Semiclass Opt, 3, 223-227(2001).

[4] W.K. Wootters, Phys. Rev. Lett. 80, 2245 (1998).

[5] A. Osterloh, L. Amico, G. Falci, et al. Nature 416, 608 (2002).

L.A. Wu, M.S. Sarandy, D.A. Lidar, Phys. Rev. Lett. 93, 250404(2004).

[6] S. Ghosh, T.F. Rosenbaum, G. Aeppli, et al. Nature 425, 48(2003).

V. Vedral, Nature 425, 28(2003).

[7] S.P. Walborn, P.H. Souto Ribeiro, L. Davidovich, et al. Nature 440, 1022(2006).

[8] B.M. Terhal, K.G.H. Vollbrecht, Phys. Rev. Lett., 85, 2625-2628(2000).

S.M. Fei, J. Jost, X.Q. Li-Jost and G.F. Wang, Phys. Lett. A 310, 333-338(2003).

S.M. Fei and X.Q. Li-Jost, Rep. Math. Phys. 53, 195-210(2004).

S.M. Fei, Z.X. Wang and H. Zhao, Phys. Lett. A 329, 414-419(2004).

P. Rungta and C.M. Caves, Phys Rev A 67, 012307(2003).

[9] F. Mintert, M. Kus, A. Buchleitner, Phys. Rev. Lett. 92, 167902(2004).

[10] K. Chen, S. Albeverio and S.M. Fei, Phys. Rev. Lett. 95, 210501(2005).

[11] K. Chen, S. Albeverio, and S.M. Fei, Phys. Rev. Lett. 95, 040504(2005).

[12] H.P. Breuer, J. Phys. A 39, 11847(2006).

[13] H.P. Breuer, Phys. Rev. Lett. 97, 080501(2006).

[14] J.I. de Vicente, Phys. Rev. A 75, 052320(2007).

[15] C.J. Zhang, Y.S. Zhang, and S. Zhang, et al. Phys. Rev. A 76, 012334(2007).

[16] E. Gerjuoy, Phys. Rev. A 67, 052308(2003).

[17] Y.C. Ou, H. Fan and S.M. Fei, Phys. Rev. A 78, 012311(2008).

[18] M. Li and S.M. Fei, Phys. Rev. A 82, 044303(2010).

[19] X.F. Qi, J.C. Hou, Positive finite rank elementary operators and characterizing entanglement of states, in press. 